\title{
La cultura skate en las sociedades contemporáneas: una aproximación etnográfica a la ciudad de Madrid
}

\author{
Skate culture in contemporary societies: an ethnographic \\ approach to the city of Madrid
}

\author{
ISRAEl MÁRQUEZ
}

Universidad Complutense de Madrid isravmarquez@gmail.com (ESPAÑA)

\section{RubÉn DíEz García}

Universidad Carlos III de Madrid y Universidad Complutense de Madrid ruben.diez@uc3m.es (ESPAÑA) ${ }^{1}$

Recibido: 31.03 .2014

Aceptado: 09.01.2015

\section{RESUMEN}

Este artículo propone un acercamiento al fenómeno del skate en la ciudad de Madrid a partir de un estudio etnográfico multisituado en el que se combina investigación online y offline. A partir de esta metodología, ofrecemos una interpretación de algunas de las principales características que presenta la cultura skate en nuestra sociedad, particularmente en la ciudad de Madrid, poniendo énfasis en los aspectos más intersubjetivos y culturales de esta realidad.

La práctica del skate en Madrid ha experimentado un visible incremento en los últimos años y el marco analítico que proponemos para el estudio de su difusión y generalización responde a cambios en el orden cultural de las sociedad occidentales y de la propia cultura skate, en dinámica interdependencia con la

${ }^{1}$ Quisiéramos agradecer a los coordinadores de este Monográfico, especialmente a Raúl Sánchez García, y a los dos revisores anónimos de la Revista Empiria su interés por nuestro trabajo. Asimismo, queremos agradecer la simpatía y amabilidad mostrada por Blake, Sergio Jungle y el resto de skaters que hemos conocido durante el trabajo de campo. 
esfera económica, como orden de producción y distribución de servicios y productos culturales y deportivos.

\title{
PALABRAS CLAVE
}

Etnografía; Cultura; Deporte; Madrid; Skate.

\begin{abstract}
This paper offers an approach to the study of skateboarding in the city of Madrid through a multi-sited ethnographic study which combines online and offline research. Based on this methodology, we show an analysis of some of the main characteristics of the skate culture in our society, particularly in the city of Madrid, putting the emphasis on the most intersubjective and cultural aspects of this reality.

In recent years skateboarding has experienced a visible increase in Madrid and the analytic frame that we propose for studying its diffusion and escalation, answers to changes in the cultural order of western societies and the skate culture in itself, in dynamic interdependence with the economic sphere, as the order of production and distribution of cultural and sporting services and goods
\end{abstract}

\section{KEY WORDS}

Ethnography; Culture; Sport; Madrid; Skate.

\section{JUSTIFICACIÓN Y METODOLOGÍA}

Nuestra propuesta de investigación se centra en el estudio de los valores, creencias y elementos constitutivos de la identidad de la comunidad de skaters a día de hoy, así como de las pautas de acción que despliegan en los diferentes espacios urbanos donde desarrollan sus prácticas, habilidades y trucos de patinaje. Asimismo, nuestro programa de investigación incorpora otros aspectos de la realidad social interconectados con el orden cultural como son las actividades de producción y distribución de los "medios" (tablas, protecciones, accesorios, infraestructuras y espacios) y "destrezas" (escuelas de patinaje, jornadas y encuentros sobre práctica del skate) que dotan a esta comunidad de una capacidad expresiva, expansiva y generadora de nuevas prácticas culturales y "estilos de pensar" (Douglas, 1988) que está teniendo un gran potencial para atraer nuevos seguidores e incrementar su visibilidad en la ciudad de Madrid.

Para la obtención de los datos relativos a esta investigación hemos recurrido a técnicas etnográficas de carácter tradicional como la observación participante y las entrevistas en profundidad. Hemos seguido, observado e interactuado 
con algunos grupos de skaters en zonas aledañas a Madrid Rio, la Plaza de Legazpi, el Parque de Arganzuela, la Cuesta de Moyano y la Plaza de Santo Domingo, así como en el SkatePark de Madrid Río o la rampa del Centro Social Autogestionado La Tabacalera de Lavapies. ${ }^{2}$ Asimismo, hemos realizado entrevistas a skaters en el contexto de la acción y en profundidad, que han sido grabadas para su posterior análisis, y hemos asistido a algunos eventos en torno a la práctica de este deporte como el Madrid Skate Film Festival en sus ediciones de 2012 y $2013 .^{3}$

Por otro lado, dada la importancia que tiene Internet y las redes sociales en la vida diaria de esta comunidad, -ya que son utilizadas para intercambiar información, comentar vídeos y otro material visual, realizar "quedadas", etc., hemos extendido la etnografía cara a cara o presencial a contextos digitales, llevando a cabo una "etnografía virtual" (Hine, 2004) o "netnografía" (Konizets, 2010).

Al combinar investigación online y offline, nuestro trabajo puede ser descrito como una "etnografía mixta" (blended ethnography), esto es, un tipo de etnografía a medio camino entre la tradicional etnografía cara a cara y la nueva "netnografía" desarrollada en plataformas digitales tipo blogs, foros, redes sociales, mundos virtuales, etc. (Ibid.). Pensamos que en una época como la actual, donde Internet y las redes sociales están tan integrados en la vida cotidiana de las personas, particularmente entre los jóvenes, la investigación online es tan necesaria como la offline si queremos entender el verdadero alcance de los colectivos que estudiamos. En especial si estos hacen un uso intensivo de estas herramientas, como es el caso del colectivo estudiado. Por eso realizamos (y seguimos realizando) observación participante y entrevistas tanto en contextos de interacción presencial cara a cara, como en espacios digitales de interacción online, que nos permiten obtener un registro de datos más amplio y un retrato más fiel y coherente de lo que realmente significa hoy ser skater, de cómo la sociedad de la información está cambiando los modos en que los skaters conciben, comentan y experimentan su práctica.

Por otro lado, esta diversidad de espacios físicos y digitales por los que los sujetos objeto de nuestro estudio se mueven y circulan cotidianamente convierten nuestra etnografía en una "etnografía multisituada" (Marcus, 1995) que no se limita a un único espacio (un barrio determinado de Madrid, por ejemplo) sino que se manifiesta en varios de ellos (diversos barrios madrileños y diversas redes digitales en Internet). Marcus propone el término "etnografía multisituada" como un modo de trascender la vieja idea de situar el trabajo etnográfico en un espacio o zona geográfica delimitada y apostar en su lugar

2 Estas etnografías han dado lugar a la elaboración de 7 Fichas o Documentos de observación. Estas son el soporte documental que ha permitido organizar y sistematizar la información obtenida durante el trabajo de campo.

3 http://madridskatefilmfestival.com. El Primer festival de cine skate de Madrid se celebró los días 1 y 2 de diciembre de 2012 y posteriormente se celebró una segunda edición durante los días 5, 6 y 7 de julio de 2013. Ambos festivales tuvieron lugar en las instalaciones de Matadero Madrid, Centro de Creación Contemporánea. 
por "examinar la circulación de significados culturales, objetos e identidades en un espacio-tiempo difuso" (Marcus, 1995: 96). Este autor reemplaza la imagen tradicional de la residencia por la del seguimiento de personas y objetos por distintos lugares, y ello por rutas que a menudo no pueden prefigurarse y a las que el etnógrafo tendrá que hacer frente con una nueva sensibilidad etnográfica de carácter más móvil y fluido. Se trata de volcar la atención no tanto en el "estar ahî" como en el "llegar ahî" (Clifford, 2008), mediante diseños de investigación y trabajos de campo que implican exploraciones en más de un lugar y que están enfocados fundamentalmente hacia el seguimiento de las conexiones y los flujos culturales, de una manera en cierto modo "coreográfica" (Janesick, 2000).

Este tipo de mirada etnográfica, -al igual que la necesidad de realizar etnografía online en un contexto en el que las tecnologías de la información juegan un papel muy significativo-, es especialmente relevante para el estudio del mundo contemporáneo, y sobre todo coherente con él, ya que si por algo se caracteriza la realidad actual es por la movilidad de personas, comunidades, culturas, objetos, narrativas, biografías, conflictos, etc., tanto en contextos físicos o presenciales como en los múltiples espacios digitales de Internet. La tarea del etnógrafo multisituado es, pues, ofrecer un retrato coherente de este panorama tan heterogéneo. Explorando para ello cómo se producen los vínculos, redes y conexiones entre personas, y cómo estas se negocian y transforman a lo largo del tiempo y en los diferentes espacios por los que se manifiestan y desarrollan. Una perspectiva que informa nuestro trabajo etnográfico, dado que seguimos a los skaters a través de distintas redes y espacios, tanto físicos como digitales.

Por último, esta mezcla de investigación online y offline (o "mixta") y de etnografía "multisituada" se propone también como una estrategia de "triangulación" (Oppermann, 2000) que permite contrastar y verificar datos procedentes de contextos diversos (tanto físicos como digitales) y aumentar así el alcance y la validez de la investigación. Todo ello con el objetivo de reflejar lo más fielmente posible la complejidad del fenómeno que pretendemos describir. Sin embargo, somos conscientes de que esta nueva sensibilidad etnográfica implica abandonar en gran medida las pretensiones holísticas, puesto que en la mayoría de los casos "es imposible dar cuenta de la interconectividad en su totalidad" (Hine, 2004: 75). La propia idea de un estudio holístico, aunque se desarrolle en un espacio claramente delimitado, es una especie de ficción intelectual, puesto que toda descripción etnográfica es irremediablemente parcial y selectiva (Hammersley y Atkinson, 1994). En este artículo presentamos los resultados de una primera 'aproximación etnográfica' a la práctica cultural del skate y por tanto una respuesta inicial y en desarrollo a su visible incremento en la ciudad Madrid. 


\section{APROXIMACIÓN A LA PRÁCTICA DEL SKATE}

Para entender la cultura skate primero debemos entender qué significa esta práctica y cómo se ha venido experimentando a través del tiempo. La práctica del skateboarding o skate consiste en utilizar una tabla con ruedas (conocida como skate o monopatín) para deslizarse por el asfalto y realizar una diversidad de trucos que van desde elevar la tabla del suelo realizando piruetas o utilizar el mobiliario urbano (bancos, bordillos, escalones, barandillas, etc.) como medio para desarrollar maniobras y deslizarse a través de él. El skate está íntimamente relacionado con el surfing y en sus orígenes se desarrolló en la costa oeste de los Estados Unidos como una alternativa a la tabla de surf para ser practicado en tierra, normalmente en piscinas vacías y otros planos inclinados donde los skaters eran capaces de simular los movimientos de las olas cuando no podían usar sus tablas de surf debido a las malas condiciones temporales. De hecho, en los orígenes del skate éste se conocía con el nombre de sidewalk surfing o "surf de las aceras" y muchas veces se practicaba descalzo o de rodillas, buscando una vez más emular la experiencia del surfing.

Podemos afirmar que el skate es básicamente un invento norteamericano, y ha sido en torno a palabras, revistas, vídeos y música norteamericanos que se ha desarrollado en el resto de países todo un imaginario sociocultural en torno a esta práctica. No hay más que observar que todo el vocabulario técnico que los skaters utilizan para comunicar sus trucos y maniobras es inglés: lip, flip, kickflip, hellflip, backflip, hardflip, grab, boneless, slide, backside, frontside, nose, tail, regular, goofy, etc. Los diferentes estilos y/o lugares que se asocian con la práctica del skateboarding también se conocen por sus nombres en inglés: bowl, half-pipe, old school, ramp, streetstyle, slalom, spot, skatepark, freestyle, downhill o longboard, mini cruiser, etc.

Esta práctica deportiva, como otros nuevos deportes en nuestras sociedades "forman parte de la producción de necesidades culturales y están relacionadas con la estructura social propia de cada contexto" (Laraña, 1986: 6). Y como muchas otras prácticas culturales, el skate surgió en California a finales de los años 50, en un contexto de desarrollo económico, social y político, en el que la tradicional ética puritana había ido perdiendo fuerza como paradigma cultural en favor de una nueva ética hedonista y consumista, en la que el placer y el juego ya habían empezado a desplazar el trabajo, la ética del esfuerzo y de la vocación, y la religión como estilos de vida predominantes (Bell, 1976, 1977). Nos situamos por tanto ante dos ámbitos diferentes pero interrelacionados, el de la cultura y el de la estructura social, entendida como orden tecno-económico. Para Daniel Bell estos dos órdenes son susceptibles de ser abordados desde esquemas conceptuales que favorecen su análisis, y facilitan al analista mostrar las contradicciones que surgen entre ambos. Si en el caso de la estructura social el principio axial que la caracteriza vendría dado por los "principios de utilidad, eficiencia (racionalidad funcional) y creciente especialización", los cambios culturales tendrían "carácter cíclico y el principio central que lo explica es la 
autorrealización del individuo, su libre expresión y autoafirmación". ${ }^{4}$ Un hecho que da lugar a importantes tensiones y contradicciones entre los principios y normas por las que se rigen la estructura social y la cultura (Bell, en Laraña 1986).

Así, por ejemplo, el skate, al igual que otros nuevos deportes como el snowboard, el surf o el windsurf, se presentan en contraposición con los deportes tradicionales y la concepción clásica de los mismos que parece más asociada a la ética del esfuerzo y de la disciplina, y con la competición como marco de la estructura de valores del capitalismo avanzado (Whitson en Beal, 1995: 253; Laraña, 1986: 9). El skate, así como su precedente el surf, su adaptación a la nieve, el snowboard, u otras variantes como el longboard, no funcionan bajo esa lógica, en la que el deporte es un medio o un instrumento para lograr mejores marcas y orientado a la competición, sino que los que lo practican lo consideran "un fin en sí mismo, cuyo valor radica en la satisfacción que produce y no en la obtención de unos resultados mensurables o en la ideología del logro" (op.cit.: 9). ${ }^{5}$

El streetstyle es quizás el estilo más conocido de skate y el responsable de que esta práctica se asocie fundamentalmente con la cultura urbana o callejera. Y ya no sólo como un deporte o cultura urbana, sino como una práctica cultural alternativa o de resistencia,-contrahegemónica-, que desafía la hegemonía de los valores capitalistas y de los deportes que lo reafirman (Beal, 1995). El streetstyle, o skate de calle, está directamente relacionado con la invención de una maniobra técnica conocida como ollie. El nombre del truco deriva de la primera persona que lo utilizó, Allan "Ollie" Gelfand, y es el truco básico del skateboarding, sobre el que se desarrollan muchos otros. Es el primer truco que un skater aprende y un paso necesario para poder desarrollar todos los demás. Este truco consiste en saltar con la tabla sin sujetarla con las manos. Al realizar un ollie, tanto la tabla como el skater saltan en el aire y permanecen en contacto durante el salto. Allan "Ollie" Gelfand consiguió revolucionar la práctica del skate con este invento, dando lugar al nacimiento del streetstyle. Gracias a este truco, el skater podía ahora saltar aquellos obstáculos que encontraba durante sus recorridos urbanos, y de hecho la práctica del skate se relacionó entonces con la búsqueda de estos obstáculos y la forma de hacer innovadores trucos

4 Queremos enfatizar el hecho de que estas afirmaciones expresan patrones de orden conceptual y hacen referencia a principios axiales que facilitan el análisis de las esferas $\mathrm{u}$ órdenes propuestos por Bell en sus obras El advenimiento de la Sociedad Post-industrial y Las contradicciones culturales del capitalismo. La introducción de estos principios axiales no busca establecer causalidades, sino destacar su centralidad o crucial importancia en cada uno de estos órdenes. Son numerosos los autores que han recurrido de forma implícita al desarrollo de principios axiales en sus formulaciones teóricas. Tocqueville lo hace en su estudio La democracia en América al destacar el proceso de igualitarización como principio que explica la difusión del sentimiento democrático en la sociedad americana del siglo XIX, o Max Weber al enfatizar la centralidad del proceso de racionalización para comprender la transformación de las sociedades tradicionales y el tránsito a otras más modernas en Occidente (Bell, 1976: 9).

5 Este hecho no implica que las personas que practican estos deportes no desarrollen un interesante afán de superación que les impulsa a mejorar su nivel de habilidades y destreza.

EMPIRIA. Revista de Metodología de Ciencias Sociales. N. ${ }^{\circ}$ 30, enero-abril, 2015, pp. 133-158. ISSN: 1139-5737, DOI/empiria.30.2015.13888 
en ellos, ya fueran bancos, bordillos, fuentes, escalones, etc. El mobiliario urbano de las ciudades se convirtió entonces en un lugar en el que los skaters podían desarrollar su imaginación y creatividad a partir de innovadores trucos y acrobacias ideados a partir de diversos elementos disponibles en la calle, inscribiéndoles nuevos usos y significados. El streetstyle es también el responsable de esa imagen estigmatizada que se ha desarrollado en torno a la figura del skater, que es visto en la percepción popular como alguien que "destroza" el mobiliario urbano con su tabla y sus maniobras, y que atemoriza a los conductores y transeúntes con su velocidad.

Con el tiempo, especialmente durante la década de los 90, el streetstyle acabó desplazando al resto de estilos y se convirtió en la imagen principal del skate a nivel internacional, algo que fue posible gracias a un mercado en expansión de material técnico, revistas, vídeos y música dominado por un modelo norteamericano que "difundió no sólo innovaciones en la práctica sino también imágenes de los mejores espacios y mobiliario urbano para patinar de muchas ciudades del mundo" (Camino, 2008: 58). Esta apropiación del espacio público por parte de la cultura skate fue visto como un problema por varios gobiernos de distintas partes del mundo, y muchos de ellos llegaron a prohibir esta práctica imponiendo multas y requisando tablas. ${ }^{6}$ Para muchas autoridades municipales y personas de a pie, los skaters representaban un estilo de vida marginal, inmaduro e improductivo que incluso dañaba la estética de las ciudades, al igual que otras subculturas entonces en auge como la del graffiti, con la que guarda varias similitudes. Otra medida para frenar la actividad de los skaters fue la instalación de los llamados skatestoppers, los cuales son pequeñas piezas de metal o cemento que se fijan en el mobiliario urbano para dificultar que las tablas se deslicen por él. Un ejemplo de estos skatestoppers lo encontramos en la plaza de la Avenida Felipe II de Madrid, colocados de tal manera que impiden a los skaters realizar su práctica en este espacio.

6 La Ordenanza de Movilidad para la Ciudad de Madrid, en su artículo 26, prohíbe expresamente "circular por la calzada utilizando monopatines, patines o aparatos similares, salvo que se trate de zonas, vías o partes de éstas que les estén especialmente destinadas, y sólo podrán circular a paso de persona por las aceras o por las calles residenciales debidamente señalizadas, sin que en ningún caso se permita que sean arrastrados por otros vehículos". http://www.madrid.es/ UnidadWeb/UGNormativas/Normativa/2005/ANM200548.pdf 

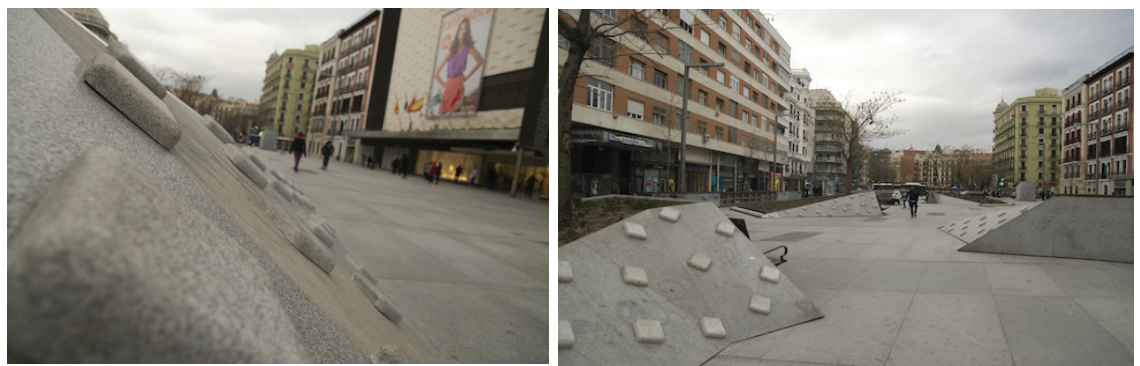

Skatestoppers en forma de pequeños bloques de cemento en la plaza de la Avenida Felipe II de Madrid Fuente: Diario Local Zona Retiro $(08 / 03 / 2011)^{7}$

\section{EL SKATE COMO SUBCULTURA}

A pesar de este tipo de estrategias institucionales para regular y controlar la práctica del skate, numerosos skaters han seguido apropiándose del mobiliario en espacios públicos, continuando esa actitud rebelde que ha caracterizado a muchos de ellos desde sus inicios y mediante la cual parece que estarían expresando su particular desafío simbólico a los modelos adultos y a las instituciones responsables de la producción y gestión oficial del espacio público. ${ }^{8}$ Esta actitud, junto con un sistema propio de creencias, valores, prácticas y comportamientos, definen al skate como subcultura, es decir, como "una unidad de funcionamiento dotada de un impacto integrado en los individuos participantes" (Gordon, 1947: 40). Según Sarah Thornton (1997), las subculturas son grupos de personas que tienen algo en común y que comparten unos mismos problemas, intereses y prácticas que los distinguen de manera significativa de los miembros de otros grupos sociales.

El aspecto subcultural del skate se manifiesta también en sus saludos y en un uso distintivo de la vestimenta, como por ejemplo el empleo de gorras, zapatillas de Nike, Converse, DC o Vans, y camisetas de marcas de skate, tales como Independent, Element, Santa Cruz, Flip, Girl, Chocolate, etc. También en la música que escuchan, normalmente estilos urbanos como el hip-hop, el punk y el hardcore. Asimismo, los skaters han desarrollado todo un vocabulario propio que les caracteriza como subcultura y que incluye expresiones como las siguientes: "skate or die" (un aforismo dentro de la subcultura skate cuyo significado es que nunca te canses de intentarlo, incluso aunque mueras en el intento), "skate \& destroy" (una frase que se difundió y popularizó a principios de los años 80 y que simboliza la naturaleza subversiva del skate y su búsqueda

7 "El Ayuntamiento 'parchea' la Avenida de Felipe II a prueba de patinadores". Disponible on-line a través del link:

http://zonaretiro.com/urbanismo/avenida-de-felipe-ii-patinadores

8 No obstante, esta caracterización, en cierto modo idealizada, admite numerosos matices. Algunos aspectos relacionados con este hecho son señalados más adelante. 
continua de espacios y mobiliario urbano para la creación de nuevos trucos), "skate is not a crime" (un eslogan dentro de la subcultura skate que se refiere a la defensa de esta práctica frente a aquellas instituciones y legislaciones que la prohíben y condenan), "Sk8" (abreviatura de skate), "pro" (abreviatura de skater profesional), "cazar un truco" (bajar desde el aire hasta el suelo con la tabla y que los pies no se salgan de la tabla cuando entra en contacto con el suelo), "planchar un truco" (caer un truco sin imperfección alguna, sin tambalearse o poner las manos en el suelo al situarse sobre la tabla), "grindar" (señala la acción de deslizarse sobre un mobiliario urbano con la tabla), etc.

Es por medio de todos estos elementos que los skaters manifiestan su particular estilo y "dramatizan la realidad", es decir, se "ponen en escena" y se hacen notar como únicos y distintos: "toda identidad necesita 'mostrarse', comunicarse para hacerse 'real', lo que implica por parte del actor individual o colectivo la utilización 'dramatúrgica' de aquellas marcas, atributos y elementos que le permitan desplegar su identidad" (Reguillo, 2000: 98-99). La siguiente imagen muestra la pierna de uno de nuestros informantes. En ella se advierten dos "marcas" o "atributos" de la cultura skate con las que este informante construye y "presenta" (Goffman, 1993) su identidad como skater: unas zapatillas Converse (una marca característica de la cultura skate) y un tatuaje de una tabla de skate o monopatín, el objeto emblemático de esta cultura, una suerte de "tótem" sobre el que los skaters construyen su práctica, su identidad y su sentido de comunidad.

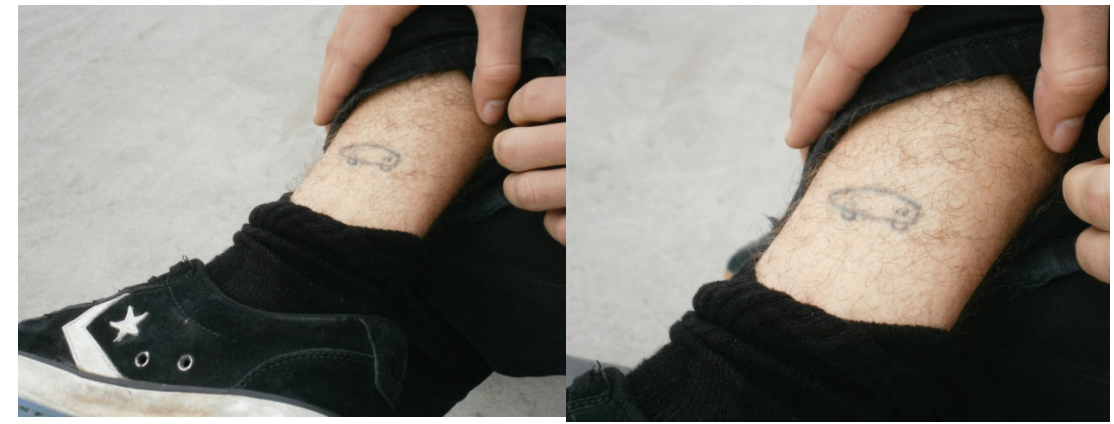

Zapatillas Converse utilizadas para la práctica del skate y tatuaje de una tabla o monopatín Fuente: Etnografía-2 (21/11/2012)

9 Entendemos el concepto de "estilo" en el sentido expresado por Hebdige (2004), esto es, como la manifestación simbólica de las culturas juveniles, expresada en un conjunto más o menos coherente de elementos materiales o inmateriales que los jóvenes consideran representativos de su identidad como grupo. 
De esta forma, los objetos simbólicos que definen la subcultura skate (el vestuario, la apariencia, el lenguaje -verbal y corporal-, las formas de interacción, la música, los tatuajes, etc.), se configuran como una "unidad junto a las relaciones, la situación y la experiencia del grupo" (Hall, citado en Hebdige 2004: 159). Surge así la construcción simbólica "nosotros los skaters", la cual establece alteridades con respecto a otras manifestaciones subculturales como los punks, los mods, los rockers, los raperos, etc. Ellos son los "otros", están fuera de la identidad skate como grupo social particular, pertenecen a otro "mundo", a pesar de todos los elementos que comparten: saludos, amistad, juventud, música, apropiación urbana, consumo de drogas, etc.:

los chicos no hablan inglés y yo no hablo español, pero no hace falta para reconocerlos y verlos [...] tú extiendes la mano así... ; todo el mundo hace esto, no hay problema, en cualquier sitio. En Estados Unidos tú vas a cualquier parque o a cualquier Estado y es universal, es la cultura y tú vienes aquí y es lo mismo [...] está asociado con el skate. Es 'cool' todos los chicos hacen así, en cualquier sitio y es simple... (ENT-1) ${ }^{10}$

en todos los lados, a cualquier sitio que vas, da igual, llegas y lo primero que pasa es que haces amigos, siempre. Yo me he ido solo de aquí a Valladolid, a una competición, sin conocer a nadie, y llegué y me hice amigos, tío. He dormido en su casa [...] Llego, saludo, y tienes una amistad. Siempre haces amigos en el skate $\left(\right.$ ENT-2) ${ }^{11}$

el tipo de gente que hace skate, somos todos parecidos, somos gente que hemos sido outsiders de la sociedad, que nos ven raro por patinar y entonces cuando éramos pocos y veías a alguien por la calle patinando, le saludabas, le conocías... Ahora ya, siendo tantos, pues ya menos, pero antes era como..., conocías a la gente [...]

de vez en cuando nos íbamos a otra provincia, a conocer otro skatepark y a conocer a la gente de allí, y claro conocías a otros skaters de otra ciudad. Entonces intercambiabas teléfonos, contactos y mantenías el contacto de una provincia a otra. Ahora es más fácil con Internet, pero antes era como que..., llamabas. Oye que vamos a ir allí siete personas y tal, te quedabas a dormir donde podias... [ENT-3] ${ }^{12}$

10 El entrevistado es un joven estudiante norteamericano que durante unos meses realizó cursos universitarios en España y junto con otros jóvenes españoles practicaba skate en instalaciones como el skatepark de Madrid Rio o la rampa de La Tabacalera. La entrevista se desarrolló en inglés y la traducción es nuestra. En adelante (ENT-1).

11 Esta es una entrevista grupal que se hizo a un grupo de skaters mientras practicaban sus trucos. Los informantes clave son básicamente dos. Uno de ellos reside en Barcelona y es de origen brasileño, pero en el momento de la entrevista se encuentra en Madrid por unos días. El otro lleva una tiempo sin practicar skate a consecuencia de una lesión de rodilla y está dentro del grupo que coordina la rampa del colectivo Cultura Skate en Tabacalera. Hay un tercer informante, pero sus intervenciones son menos relevantes y no practica skate. En adelante (ENT-2).

12 El entrevistado es un skater con muchos años de experiencia y bien conocido dentro de la escena madrileña. En adelante (ENT-3). Pertenece a diversos colectivos relacionados con la 
Sin embargo, a pesar de querer mantener su diferencia y singularidad, las subculturas nunca son "puras" y homogéneas, y el skate no es una excepción. Algunos autores y analistas de las culturas juveniles y musicales (Clarke, 1990; Thornton, 1995) han criticado la tendencia a minusvalorar la heterogeneidad de posiciones y la competencia dentro de una subcultura en cuanto a motivaciones, prácticas o ideas, así como en lo referente a la idealización de determinadas prácticas de resistencia. Esta heterogeneidad o diversidad intra-subcultural emerge en el caso del skate si centramos el foco de análisis en elementos y aspectos como, por ejemplo, la posición social, el nivel de destreza de los skaters, el tipo de variedades, habilidades y trucos susceptibles de ser practicados, la indumentaria, los gustos musicales, o determinados hábitos de comportamiento asociados con la cultura skate:

si hablas con gente de mi edad, te dirán que patinan todo, yo patino todo, porque es lo que me gusta. Me gusta tanto la rampa, como patinar por la calle, incluso el longboard también. Practico todo lo que pillo. Pero si hablas con un chaval joven, normalmente le gustará más el street. O le gusta lo que tiene más a mano, o lo que le gusta hacer mejor [...] Yo cuando empecé a patinar, las rampas no las tenía a mano y empecé con el street. Incluso antes de hacer trucos de street yo ya iba por la calle, pues no sé, por ejemplo a comprar el pan, o hacer los recados... e iba con el patín todo el rato [...]

Yo en Venezuela por ejemplo lo viví mucho. Yo patinaba con el hijo del embajador de Alemania y con un traficante de drogas... [risas] en el mismo grupo y patinando todos en el mismo grupo (ENT-3)

yo patino porque me gusta mucho, pero hay mucha competición entre la gente, sabes. Siempre hay muchos chavales que te ven hacer un truco, y lo quiere hacer mejor. Eso siempre, en cualquier lado [...] Yo siempre voy de buen rollo y si veo que lo acepta, o lo hace mejor que yo... [...] Siempre aprendes de la gente que práctica mejor que tú. Yo ahora mismo, con ese chaval, le estaba enseñando... (ENT-2)

[hay] gente que le gusta patinar en la bowl o [gente que] prefiere patinar en la calle [...] para las zapatillas, pues zapatillas de skate, seguro. Las zapatillas si pueden ser importantes, pero las ropas son algo extra, tú no tienes que... yo veo chicos que realmente les gustan las ropas de skate, pero luego visten normal; no es necesario vestir de un modo concreto [...] no puedo mentir, yo cuando era más joven era más así, pero ahora... yo bebo cerveza cuando estoy patinando, pero eso es todo. Pero sí, está asociado con la cultura, tú vas a cualquier sitio y ves gente con porros... absolutamente. Yo no tengo problema con nadie porque esté fumando, está bien... pero cuando yo estoy patinado por la calle, la gente mayor te mira como si fueras un pedazo de mierda y eso no es justo, no todo el

práctica del skateboarding y el longboard como la escuela de skate y longboard Entabla (http:// www.entabla.es) y 80Brigade, "un grupo de skaters fundado en Madrid en 2009 (antes MOSS Brigade) por varios amantes del skateboarding. Es una organización abierta a todos los interesados en el movimiento skater. La diversión y el aprendizaje, son sus principales metas" (http://www. entabla.es/80b). 
mundo es así porque esté asociado con eso (ENT-1)

Las subculturas interactúan y reciben influencias de otras subculturas. Por ejemplo, en el caso del skate es muy notable la influencia de la música hip hop y el uso de ropa holgada en los denominados "skater-raperos": "hay de los dos estilos, del estilo punk y del estilo raper" (ENT-2). Por otro lado, la subcultura punk ha ejercido una notable influencia en los llamados "skaterpunks", los cuales visten pantalones estrechos, colores oscuros y escuchan música punk, metal y hardcore. En la década de los 90 era común asociar a los "skater-raperos" con la práctica del streetstyle, y a los "skater-punks" con el skate vertical practicado en skateparks, rampas y half-pipes, aunque tal división se ha vuelto más compleja durante los últimos años, y es posible encontrar "skater-punks" que practican el streetstyle y "skater-raperos" que practican el skate vertical. Ambos casos ponen de manifiesto no sólo la influencia de otras subculturas en la subcultura skate sino la existencia de subgrupos dentro de la misma que han sido formados en torno a representaciones y actitudes recibidas de otras subculturas, el punk y el hip hop, respectivamente. ${ }^{13}$ El skate revela así su condición de híbrido subcultural, de "bricolaje" (Lévi-Strauss, 1970), como en realidad lo es cualquier subcultura, pues en todas ellas se produce una mezcla o fusión de varios elementos que dan lugar a nuevos significados:

cuando el bricolador cambia el objeto significante de lugar y lo emplaza en una posición distinta dentro de ese discurso, empleando el mismo repertorio global de signos, o cuando el objeto es resituado en un conjunto totalmente distinto, se crea un nuevo discurso, un mensaje distinto se transmite (Clarke, citado en Hebdige 2004: 144)

Al igual que otras subculturas como el punk y el hip hop, y otras como la subcultura mod, rave o grunge, el skate ha acabado siendo mercantilizado e integrado a la cultura hegemónica, desactivando en el proceso algunos de sus significados subversivos. Si inicialmente, cuando nacen, las subculturas representan el "ruido" (en contraposición a la "armonía"), Hebdige (2004: 130) habla de dos procesos fundamentales a la hora de "reconstruir" y de "reintegrar" a las mismas: i) la conversión de signos subculturales (vestuario, música, etc.) en objetos producidos y distribuidos en masa, esto es, la forma mercantil propia del orden tecno-económico (Bell, 1977); y ii) el "etiquetamiento" y redefinición de la "conducta desviada" por parte de los grupos dominantes: las autoridades públicas, la policía, los medios de comunicación o el sistema judicial (la forma ideológica).

Respecto a la forma mercantil, toda nueva subcultura establece nuevas

13 La importancia de músicas como el hip-hop o el punk en la subcultura skate y la formación de subgrupos en torno a ellas pone de manifiesto el hecho de que las subculturas y estilos juveniles tienden a ser subculturas musicales. A través de la música determinadas personas consiguen reconocerse a sí mismos como grupos, como una organización particular de intereses individuales y sociales, de mismidad y diferencia (Hebdige, 2004; Frith, 1996). 
tendencias, genera nuevos estilos que retroalimentan las correspondientes industrias (pensemos en el beneficio que industrias como la textil o la maderera han obtenido con la extensión de la cultura skate, el surf o el snowboard). Tan pronto como las innovaciones originales de la subcultura se traducen en mercancías al alcance de todos, quedan "inmovilizadas", arrancadas de sus contextos originarios por las respectivas industrias y vueltas comprensibles. Así, lo que en un principio fue un reto simbólico se convierte, tras su mercantilización, en algo convencional, generando nuevas mercancías, nuevas industrias o rejuveneciendo las antiguas. Marcas como Vans, Santa Cruz, Element, Independent o Etnies, han contribuido a la mercantilización de la imagen del skater a nivel global dirigiéndose no sólo a los practicantes de esta actividad sino a jóvenes pertenecientes a otros estratos socioculturales, quienes se han dejado seducir por la estética de estas marcas y sus atractivos logotipos aunque no se consideren a sí mismo skaters ni practiquen esta actividad.

Por otro lado, mediante la forma ideológica los grupos dominantes etiquetan y redefinen las culturas juveniles, negando su voluntad subversiva unas veces (mediante el recurso al exotismo o al espectáculo), y exagerando las diferencias otras (presentándolas como conductas desviadas, problemas sociales o una amenaza para la sociedad). La práctica del skate es presentada unas veces como un espectáculo exótico practicado por unos jóvenes que visten pantalones anchos y que saltan bordillos y bancos de una manera llamativa. ${ }^{14}$ Otras veces se presenta como un problema social que pone en peligro a conductores y transeúntes y que daña la propia estética de las ciudades con su "destrucción" del mobiliario urbano y los edificios públicos. Los grupos dominantes buscan entonces diferentes estrategias o formas de redefinir esos usos desviados normalizándolos o formalizándolos según sus intereses. Una de las estrategias más visibles de este tipo de normalización es la construcción de skateparks, espacios donde los skaters pueden ser vigilados y controlados más eficazmente.

\section{LOS SKATEPARKS O LA NORMALIZACIÓN DEL SKATE}

Siguiendo este punto de vista, la apuesta de las autoridades municipales por la construcción de skateparks en numerosas ciudades y municipios de España (como Madrid, Barcelona, Leganés, Paterna o Reus, por citar sólo algunos) y en las principales ciudades de diferentes países a lo largo del mundo, puede interpretarse como un proceso de "regularización", "formalización" o "racionalización" de su práctica. No obstante, los skateparks primigenios como hemos aludido anteriormente, fueron introducidos y promovidos por los primeros grupos de profesionales dedicados al skate como una forma de ganarse

14 Así por ejemplo, bajo el auspicio de las autoridades municipales se ha promovido en el Distrito de Villaverde el taller "San Cris se mueve", un taller de skate en el que se ayuda a los jóvenes a mejorar su técnica de patinaje (http://rollinsancris.files.wordpress.com/2013/04/publirollin.jpg), o en las instalaciones de Matadero Madrid se dio acogida al Skate Film Festival. 
la vida y obtener ingresos económicos. La iniciativa de estos profesionales dio lugar a un incipiente mercado en torno al skate y su práctica en Estados Unidos en los años setenta y ochenta ${ }^{15}$ que posteriormente se ha desarrollado como un mercado de producción y distribución muy extenso y diversificado: tablas, accesorios, indumentaria, revistas, filmes y documentales, cursos y escuelas de patinaje, diseño y construcción de skateparks, -incluso una "universidad del skate" tal y como nos señalaba uno de nuestros informantes clave (ENT-3). ${ }^{16}$

Los skateparks se empiezan a construir principalmente durante la segunda mitad de la década de 1970 en Estados Unidos coincidiendo con el auge del skate como nuevo pasatiempo juvenil. Los skateparks pueden entenderse como parques temáticos para la práctica del skate, es decir, simulaciones de piscinas vacías o pools, rampas, planos inclinados y todo tipo de mobiliario urbano (bancos, barandillas, bordillos, etc.). La construcción de skateparks se debe a la iniciativa de compañías de skate, empresas y ayuntamientos, y el objetivo de su impulso por parte de las autoridades municipales parece ser el de controlar y regular la práctica del skate (especialmente su modalidad más problemática, el streetstyle) otorgando un espacio reglamentado para ello. ${ }^{17}$

Algunos skaters han sentido cierta hostilidad hacía los skateparks pues los ven como sustitutos artificiales de la calle, como sitios cerrados donde "encerrarlos" y "recluirlos" para que así no deterioren el mobiliario urbano de la ciudad, ya que la práctica del skate en calle presupone todo un desafío a los usos, mecanismos y formas tradicionales de la vida urbana (Borden, 2001). Estos skaters encuentran limitados los skateparks frente a la libertad de acción que ofrece la ciudad, donde cualquier elemento urbano es susceptible de una nueva apropiación, de un nuevo truco o deslizamiento con la tabla (Ibid.) y en este sentido lo expresan al afirmar que "el espíritu del skate está en la calle" (ENT-2):

- El otro día, llevaba mucho tiempo sin ir a patinar a la calle, porque siempre he estado por aquí [el skatepark], por el curro y tal...; y el otro día fui ahí al Palacio de Congresos y, ¡buah!, volvía otra vez a mi vida, sabes...

- Se echa de menos eso...

- Y a veces sueño, de ir por la calle, me he agarrado al autobús, he pasado entre la gente, en Sol, que estaba así, y entre la gente patinando; eso es lo mejor de todo... [...] hay muy pocos ahora en la calle...

15 Este hecho se pone de manifiesto en el documental de Stacy Peralta 'Bones Brigade: An Autobigoraphy' (2012), que cuenta la historia y las experiencias del grupo de skaters 'Bones Brigade'. Este equipo creado en 1979 revolucionó y reinventó la práctica del skate en los ochenta. http://bonesbrigade.com.

16 http://www.bryggerietsgymnasium.se/in-english.

17 En el caso particular de la ciudad de Madrid, el vicealcalde de la ciudad señalaba durante la inauguración del skatepark de Tetuán: "La pasión por este deporte, que para muchos jóvenes se extiende a un estilo de vida, es patente en nuestra ciudad. Y era necesario compatibilizar la práctica segura de esta afición con el resto de los usos tradicionales que se realizan en los espacios públicos" (http://www.espormadrid.es/2009/11/skate-urbano-en-tetuan.html). 
- Y a parte por las leyes también, se ha endurecido bastante. Antes por ejemplo te daban el toque, 'eh!', no patinéis aquí, y tal, era el rollo. Ahora, a la mínima que..., este, pacá, documentación y te ponen la multa... (ENT-2)

están los skatepark, que ponen zona de street, pero eso es como..., para un skater un skatepark, puede que..., en una temporada, y te motiva algo, pero no del todo. Porque tú quieres ir por la calle y descubrir nuevos sitios. La gracia del street es que tú encuentres un sitio y lo domines, ¿no?, como domar a un caballo. En plan, mira, he encontrado una barandilla, y empiezas a hacer cosas en la barandilla. Me he encontrado un bordillo que está muy bien, pues vas allí y hasta que te sale todo (ENT-3)

Sin embargo, esta no es una actitud radical que compartan el conjunto de los skaters, ya que son mayoría los que demandan y usan este tipo de instalaciones para disfrutar de su práctica y para mejorar sus técnicas de patinaje o que lo alternan con la práctica del street en diferentes lugares diseminados por la ciudad. Por otro lado, muchos de nuestros informantes hacen notar su hastío debido a la dificultad de poder disfrutar cómodamente y de forma segura de las instalaciones, a consecuencia de los horarios de las mismas y su alumbrado (ENT-2) y de la alta ocupación que algunos skateparks experimentan en determinadas horas del día, épocas del año o en fines de semana, dado el progresivo aumento de personas que acuden a algunas de estas instalaciones y que practican skate, patinaje (rollers y patinetes o scooters) o $\mathrm{BMX}^{18}$ en ellas:

- Ha venido mucha gente nueva, los de siempre, sabes, la gente de Pasillo Verde, Colón, Opera y Goya... y luego ya muchos críos, muchos niños peques, mucha gente nueva...

- Es la putada, los domingos sobretodo es cuando esto se desmadra (ENT-2)

para mí un artilugio son los scooters, estos... los del manillar [...] Aparte es que eso sí que jode mogollón. Si veis el tubo de..., el coping, el tubo de metal [que rodea y delimita una bowl] está lleno de marquitas del scooter, claro, hacen así... y claro eso tiene un..., que no es plano, sino que tiene dos pequeños filos [y al golpear] eso va doblando el tubo [...] Los BMX a mí particularmente no me molestan demasiado, me molesta el típico que se mete con bicicleta de montaña, que está paseando, que no sabe muy bien las líneas que estás tomando... Pero el $B M X$ que ya sabe rodar es más respetuoso que ese tipo de gente, que el que va de dominguero o va paseando... Yo tengo un amigo que se fracturó las dos piernas por culpa de un tío que iba con una bici de montaña. Un niño que iba con una bici de montaña en un skatepark en La Coruña... (ENT-3)

El fenómeno de la importante y visible construcción de skateparks como actuación urbanística patrocinada por las propias autoridades municipales se orienta, por tanto, hacia la regulación y el control de su práctica en las ciudades,

18 Bike / Bicycle Moto Cross.

EMPIRIA. Revista de Metodología de Ciencias Sociales. N. ${ }^{\circ}$ 30, enero-abril, 2015, pp. 133-158. ISSN: 1139-5737, DOI/empiria.30.2015.13888 
y como una alternativa al streetstyle y a su práctica en los lugares, -o spots-, diseminados en el espacio público de la ciudad cuyo mobiliario o configuración es propicia para practicar sus trucos y maniobras. Un hecho que ha favorecido la creación de un interesante mercado de microempresas y empresas que diseñan y construyen este tipo de instalaciones, y que van desde los skateparks más respetados por la calidad de sus instalaciones, hasta los que cuentan con menor prestigio entre los skaters, ya que su configuración y calidad no es tan propicia, pero que en ocasiones cuentan con el favor de las instituciones municipales dado que el coste de construcción es más ajustado que el de los más prestigiados por los skaters.

En cualquier caso, la incentivación y la promoción por parte de las instituciones de una práctica controlada y regulada del skate en este tipo de instalaciones en ciudades como Madrid encuentra en numerosas ocasiones derivas por parte de los skaters que siguen practicando y alternando el streetstyle con la práctica en este tipo de instalaciones. De modo que es común encontrarse en las zonas cercanas a estas instalaciones grupos de skaters que practican sus trucos favoritos en los spots más tradicionales y clásicos del barrio y en nuevos spots que han surgido tras las transformaciones urbanísticas más recientes, como por ejemplo la Plaza de Santo Domingo, Cuesta de Moyano o las zonas aledañas a Madrid Rio, en el caso de Madrid.

\section{LAS CONTRADICCIONES CULTURALES DEL SKATE COMO PRÁCTICA DEPORTIVA MAINSTREAM}

La progresiva difusión y mercantilización del skate ha tenido sin lugar a dudas un efecto sobre el cambio de su significado cultural y en las formas en que este se practica, con nuevas modalidades derivadas de la original, como el longboard, que tiende más hacia prácticas íntimamente relacionadas con el ocio y al disfrute y menos con el desarrollo de complicadas técnicas de patinaje. La práctica del skate, -como otras afines a él-, guarda una estrecha relación con la esfera del orden tecno-económico o de la estructura social (Bell, 1977). Aquel mercado embrionario en torno al skate, a día de hoy, se ha desarrollado extraordinariamente a nivel mundial y ha impulsado cambios en sus valores, que ahora pueden perfectamente encuadrarse en el marco de las heterogéneas preferencias y gustos que predominan en la cultura popular mainstream de la juventud de las sociedades occidentales con acceso a crecientes servicios y productos culturales y deportivos. Pero hay que tener en cuenta que este desarrollo no ha sido lineal y uniforme, sino que ha presentado "oleadas" estrechamente relacionadas con la adaptación de su práctica a los ciclos económicos, con los avances tecnológicos aplicados a las tablas y con los procesos de institucionalización y deportivización del skate (Camino, 2008: 56-57). Así por ejemplo, uno de nuestros informantes clave señalaba que se han dado "varias oleadas de modas":

EMPIRIA. Revista de Metodología de Ciencias Sociales. N. ${ }^{\circ}$ 30, enero-abril, 2015, pp. 133-158. ISSN: 1139-5737, DOI/empiria.30.2015.13888 
de repente ha habido un skate, que todo el mundo hace skate, sale en todos los anuncios y de repente un bajón. Y estamos viviendo una tercera [ola], que está subiendo otra vez, pero antes era... Había una época que llegabas a una plaza y eras tú, otro, y todos los demás rollers, y estabas tú solo ahí, porque era la oleada roller (ENT-3)

La mirada etnográfica que proponemos en torno a la cotidianidad del skate en la ciudad de Madrid, muestra tendencias y dinámicas a primera vista contradictorias, pero que en su interdependencia dan un sentido propio y actual a esta práctica. Por un lado, el incremento de la práctica del skate en Madrid y su área metropolitana, sobre todo a partir de la transformación urbanística de algunas zonas de la ciudad, -como la Cuesta de Moyano y la Plaza de Santo Domingo (2005-2007) o el paseo Madrid Rio (2006-2011)-, y la habilitación de nuevos y numerosos skateparks como los de Móstoles (2003), San Cristóbal de los Ángeles (2004), Getafe (2005), Tetuán (2009) o Arganzuela-Madrid Rio y Leganes- $\mathrm{P}^{\mathbf{o}}$ de la Chopera (2011), entre muchos otros, ha permitido la formación de espacios que funcionan como importantes puntos de encuentro y socialización para los skaters, pero también para muchos otros jóvenes que practican otros deportes (patinaje, BMX), y otros que no practican ningún tipo de deporte en estos skateparks, pero que acompañan a sus amigos a las instalaciones.

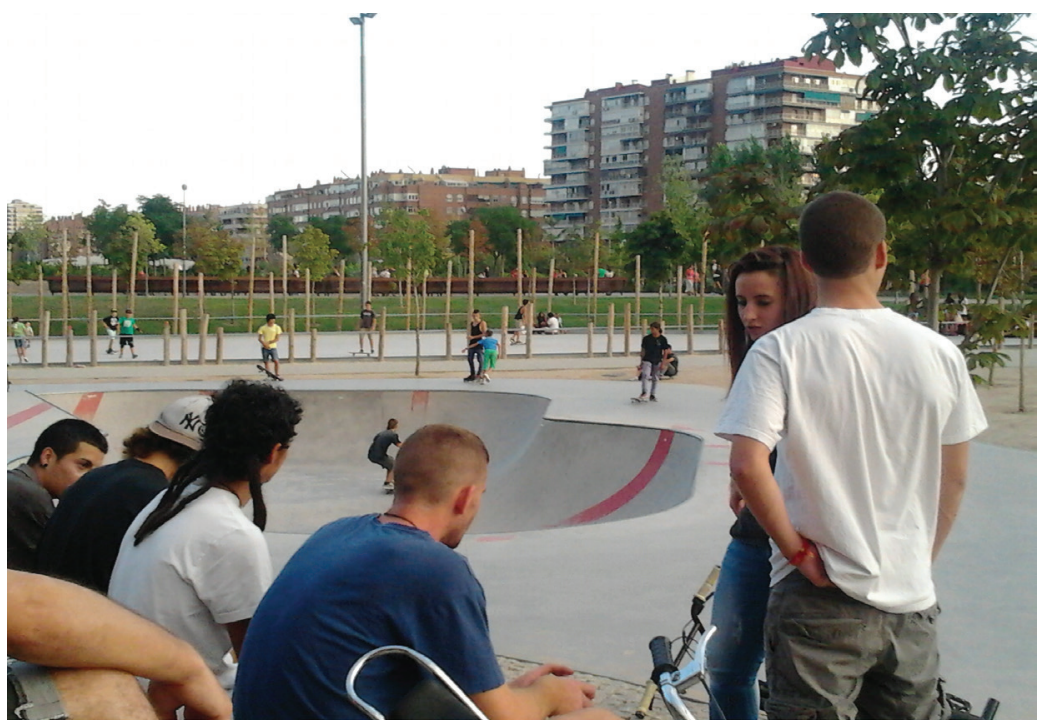

Jóvenes skaters reunidos en el skatepark de Arganzuela - Madrid Rio Fuente: Etnografía-7 $(10 / 09 / 2013)$

En una dirección parecida apunta Xavi Camino (2012: 18-19) al asociar las transformaciones urbanas que experimentó la ciudad de Barcelona en las últimas décadas, "caracterizadas por la proliferación de espacio público y la calidad del diseño urbano", con el desarrollo inesperado del fenómeno skater en la ciudad. 
Sin embargo, en el caso de Madrid y su área metropolitana hemos observado que no se trata únicamente de la proliferación de espacios públicos cuyo diseño los hace muy propicios para la práctica del skate, ${ }^{19}$ sino que además de este hecho se han construido numerosas instalaciones "formales" u "oficiales" en forma de skateparks que regulan su práctica al confinarlo en un espacio concreto, sujetos en ocasiones a horarios de uso. Todo un proceso que ha tenido lugar al auspicio de la especulación inmobiliaria y de las importantes inversiones públicas en planes de desarrollo y transformación urbanística de la primera década del siglo.

Por otro lado, como hemos aludido anteriormente, estos espacios se configuran como lugares potenciales para generar "conflicto" (Simmel 2000) en relación a la propia práctica del skate y otras más o menos similares, como el patinaje, el longboard o el BMX, que también se desarrollan en estos espacios y sus inmediaciones. Asimismo, la tensión y el conflicto pueden surgir en otras zonas urbanas al margen de las instalaciones "oficiales" (skateparks), en espacios públicos apropiados informalmente por los skaters en los que la práctica de esta actividad es susceptible de ser percibida por los transeúntes, o por los practicantes de otros deportes menos "agresivos", como un fenómeno invasivo y generador de inseguridad, pero que devuelve a la comunidad ese sentido transgresor que la práctica del skate tuvo en sus orígenes.

Una práctica habitual en este sentido es la de realizar recorridos en longboard o skateboard por la ciudad sorteando diversos tipos de obstáculos y mobiliario urbano, tales como bordillos, aceras, bancos, barandillas, farolas, etc., o por el mero hecho de disfrutar de la sensación de surfear la ciudad. ${ }^{20}$ Estas prácticas organizadas en ocasiones por empresas que distribuyen productos relacionados con la práctica del skate, pero también en ocasiones para reivindicar el derecho a patinar en la calle, suelen grabarse en vídeo y compartirse a través de Internet y de redes sociales como una forma de dejar inmortalizadas la experiencia, y sus habilidades o trucos, de modo que otros skaters puedan visionarlo para emularlo después.

Así por ejemplo, algunas de las prácticas relacionadas con el skate -en especial aquellas en las que la ciudad y el espacio público se experimentan como lugares donde no se sabe lo que uno puede encontrarse-, guardan ciertas similitudes con las prácticas espaciales de los surrealistas y los situacionistas, quienes deambulaban por la ciudad sin un objetivo concreto, buscando dejarse sorprender por el azar de lo que encontraban. Sólo así era posible descubrir realmente la ciudad y los diversos elementos y experiencias que la componen, más allá de los mapas y otros dispositivos oficiales de recorrido urbano. Una serie de "tácticas", como diría Michel de Certeau (1988) que dotan de nuevos usos, ideas y significados propios a las "estrategias" de diseño de las compañías,

19 Bordillos, rampas y escaleras como las de la Cuesta de Moyano, la Plaza de Santo Domingo o diferentes zonas aledañas al paseo de Madrid Río.

20 Este tipo de práctica recibe el nombre de cruising y pueden visualizarse numerosos ejemplos a través de YouTube: cruising reivindicativo celebrado en Madrid en 2011(http://youtu. be/d-UT1LGSu2w), longboard cruising, Madrid 2013 (http://youtu.be/taO3HXsByWQ) o New Year cruising, Madrid 2014 (http://youtu.be/MOU5PhQqygw) 
empresas, instituciones o gobiernos, los cuales intentan imponer un conjunto de normas de ideas, intereses y deseos sobre cómo debemos relacionarnos y vivir con los objetos que fabrican (los mapas de una ciudad y sus itinerarios preestablecidos, por ejemplo).

El skate, como el longboard, y al igual que su predecesor, el surf o la variante del snowboard, informan por tanto acerca de una forma de relacionarse con el medio en que se desarrolla (Laraña, 1986), -ya sea el mar, la nieve o la ciudad- que reporta al que lo practica una serie de emociones y sensaciones que le permiten obtener determinadas cotas de satisfacción y autorrealización ${ }^{21}$, -el principio axial que constituye la cultura moderna-, y para cuyo logro no existen fronteras a la experiencia, de modo que nada está prohibido y todo debe ser explorado (Bell, 1977). Sin embargo, su difusión y generalización pasa necesariamente por la comercialización y mercantilización del mismo a través de los mecanismos de producción y canales de distribución propios del orden tecno-económico de las sociedades contemporáneas, que operan con criterios de rentabilidad, productividad, eficiencia o utilidad; así como por la regulación y el control de su práctica en el espacio urbano, mediante dispositivos como los skateparks, que paradójicamente al tiempo que sirven a este fin, aumentan su popularidad y contribuyen al desarrollo de su práctica.

\section{EL SKATE EN LA SOCIEDAD DE LA INFORMACIÓN}

Como hemos señalado en el apartado anterior, muchos recorridos y trucos de skate son grabados por sus propios protagonistas como una forma de inmortalizar la experiencia y compartirla con los demás. La cultura skate ha venido realizando este tipo de documentación (audio)visual desde sus mismos orígenes, a través de tecnologías como la fotografía o el vídeo. El fenómeno, por tanto, no es nuevo, pero sí lo es el modo en que se manifiesta actualmente. El skater actual dispone de todo tipo de medios y tecnologías para grabar y hacer circular sus prácticas y experiencias, desde cámaras de fotos, hasta cámaras de vídeo o teléfonos móviles. Los skaters graban su experiencia por medio de estas tecnologías y después la comparten rápidamente a través de Internet y las redes sociales. Es esta variedad de dispositivos tecnológicos y la facilidad de hacer circular las grabaciones que realizan a partir de ellos lo que sí es nuevo en la cultura skate actual, algo facilitado enormemente por las nuevas tecnologías digitales y las herramientas de la web 2.0.

Durante nuestro trabajo etnográfico fuimos rápidamente conscientes de este hecho y creemos que es uno de los principales hallazgos de nuestra investigación: la importancia de las tecnologías digitales para la producción,

21 Dan buena cuenta de ello los videos que mostramos a continuación: http://vimeo. com/69489601; https://www.youtube.com/watch?v=do6mvcj1Srk; https://www.youtube.com/ watch?v=Iflwt55U29A; https://www.youtube.com/watch? v=uThD0Y51XfI; http://vimeo. com/13359521. 
difusión y recepción de la práctica del skate. Es por eso que decidimos extender la interacción cara a cara a la interacción digital, llevando nuestro trabajo etnográfico de las calles de Madrid al universo de Internet a través de la observación participante en estos espacios digitales (en especial la red social Facebook y páginas web específicas como culturaskate.org) y entrevistas en línea con sus usuarios.

La red social Facebook juega un papel fundamental entre los skaters. En ella, los distintos miembros de la comunidad preparan "quedadas", comparten vídeos y fotografías sobre trucos y técnicas de skate, promocionan eventos relacionados con esta práctica, etc. Facebook sirve también como plataforma de reunión de las distintas webs y colectivos relacionados con el skate en Madrid (y en España en general). Un ejemplo significativo es 'Cultura Skate', un colectivo de skaters que han creado el primer skatepark indoor de Madrid aprovechando las instalaciones de Tabacalera. Tal y como se autodescriben en su página de Facebook.

\#culturaskate es un colectivo compuesto por aficionados al monopatín que se ha creado en el recinto de la antigua Tabacalera del barrio de Lavapies, en el centro de la capital de España, Madrid. Desde el mes de Abril 2010, están fabricando un skatepark indoor (pista de skate cubierta) utilizando material reciclado y toda la fuerza humana disponible en el CSA (Centro Social Autogestionado) Tabacalera.

El colectivo no está sólo presente en Facebook sino que cuenta con perfiles en otras redes sociales como Twitter o Instagram y con una página web propia (culturaskate.org). Sin embargo, Facebook es la página principal para conocer las últimas noticias y novedades, tal y como señalan en su página web:

Por favor, mirad nuestra página en Facebook para conocer las últimas noticias de eventos, cursos y lo más importante: aperturas. ¡Gracias!

A través de su perfil de Facebook, el colectivo 'Cultura Skate' da a conocer los horarios de apertura del skatepark indoor, así como todo tipo de cursos, talleres y eventos relacionados con el skate. Asimismo, en su perfil van apareciendo diariamente diferentes vídeos, fotografías y noticias sobre skate que son un modo de mantener unida a la comunidad y de que ésta participe enviando sus comentarios, opiniones y likes, y compartiendo lo que allí se publica en sus propios perfiles y en otras redes. El colectivo también comparte temas de actualidad a medida que estos van apareciendo, en especial aquellos que suponen una crítica o prohibición a la práctica del skate. Por ejemplo, con motivo de la publicación del Proyecto de Ley de Seguridad Ciudadana de noviembre de 2013, por el que se sancionará a quienes practiquen deporte en espacios públicos no habilitados, el colectivo publicó el siguiente texto en su perfil de Facebook: 
El proyecto de Ley de Seguridad Ciudadana sancionará a quienes practiquen deporte en espacios públicos no habilitados!

\section{\#WTF \#FAIL}

Una vez más, nos vienen con nuevos pretextos para recaudar lo que sea y robar al pueblo con nuevas categorías de multas y hacer que el monopatín sea una práctica aún más en peligro por su marginación...; ;

http://www.consejo-colef.es/noticias/consejo-general/886-el-proyecto-de-leyde-seguridad-ciudadana-sancionara-a-quienes-practiquen-deporte-en-espaciospublicos-no-habilitados.html

Parece mentira, esta gente no entiende que sus propios hijos podrían engancharse al \#skateboarding tanto o temprano?

Que harán entonces?

(Por cierto, no solo el skater puede ser multado, también en bici o scooter, y roller etc...)

Desde \#Culturaskate rogamos que esta decisión estúpida se hable debidamente con el pueblo, cuanto antes!

Y que se abren más skateparks!

Y encima quieren cerrar la pista de \#Mostoles?

Mientras, vosotros, informad a vuestros padres que os apoyen!!!

Porque...

Skateboarding is not a crime!

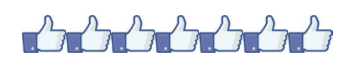

Como vemos, el texto está impregnado del tipo de lenguaje propio de Internet y la web 2.0, con símbolos como los famosos emoticonos, acrónimos como WTF, hashtags, o los iconos de "like" o "me gusta" típicos de Facebook, lo cual pone de manifiesto que este lenguaje y sus signos característicos han sido incorporados y reapropiados por la cultura skate. Este texto venía acompañado de la siguiente imagen, la cual remite a uno de los lemas clásicos del mundo del skate: "Skateboarding is not a crime" ("El skate no es un crimen"): 


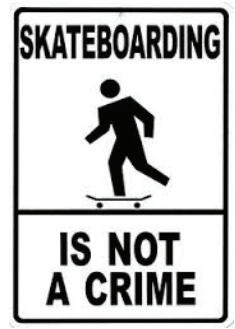

\section{Skateboarding is not a crime Fuente: Cultura Skate / Facebook}

Los skaters de la sociedad de la información no sólo utilizan las redes sociales para estar en contacto y compartir todo tipo de actividades, noticias, preocupaciones y pensamientos, sino que también están recurriendo a otro tipo de plataformas digitales propias de la web 2.0. Por ejemplo, en el momento de escribir este artículo se encuentra en marcha en la plataforma Verkami un proyecto de crowdfunding o "micromecenazgo" para financiar un documental sobre skate. El crowdfunding, también conocido como "financiación masiva" o "micromecenazgo", es la cooperación colectiva de varias personas a través de Internet con el objetivo de conseguir dinero u otros recursos para iniciar un proyecto. El proyecto se concibe como una mezcla de road movie y falso documental que utiliza el skate como hilo conductor y que de una web-serie de 10 episodios pasará a ser un largometraje. El colectivo 'Cultura Skate' se hizo eco de este proyecto en su página de Facebook y el proyecto está próximo a recibir los $6.000 €$ que necesita para llevarse a cabo. El hecho de que 'Cultura Skate' anime a la comunidad a participar en este proyecto desde su página de Facebook revela un hecho fundamental de la cultura digital actual: la conexión e interdependencia entre las distintas redes y platafomas de la web 2.0, algo necesario para alcanzar una mayor presencia y visibilidad.

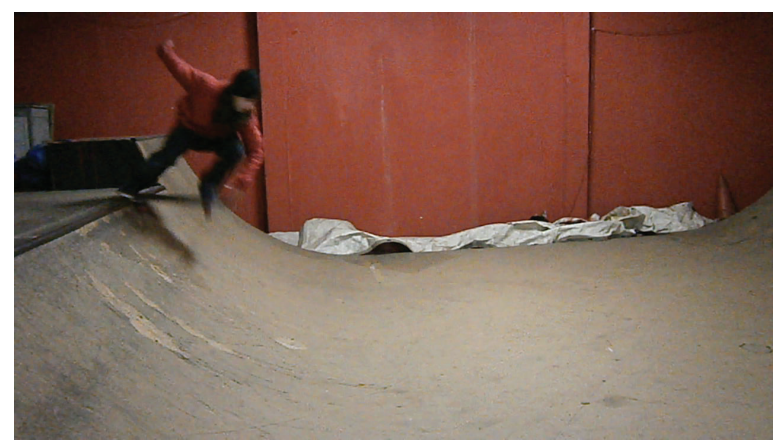

Skater en la minirampa del CSA La Tabacalera gestionada por Cultura Skate Fuente: Etnografía-5 (18/12/2012) 
Queda claro, pues, la importancia de las plataformas digitales en la cultura skate actual. Los skaters tienen actualmente una ingente cantidad de medios y redes en las que estar en contacto y compartir, comentar y participar activamente en todos ellos. Así como las cintas de vídeo, las películas y las fotografías jugaron un papel fundamental en la anterior generación de skaters, la nueva generación nacida con la cultura digital dispone de Internet y las diversas plataformas de la web 2.0 para relacionarse y afianzar su sentido de comunidad por medio de la presencia y participación activa en estas plataformas. Los skaters no sólo se relacionan cuando patinan y están juntos en la calle o en los skateparks, sino que al llegar a casa se conectan a las diversas redes que pueblan el ciberespacio y continúan esas relaciones e interacciones de manera digital. No es que estas redes y plataformas sean un sustituto a la interacción cara a cara, son más bien un complemento (Woolgar, 2005), una forma más de estar en contacto y afianzar el sentido de comunidad más allá de un contexto presencial ${ }^{22}$. En este sentido, el skate actual es tanto físico como digital ${ }^{23}$.

\section{CONCLUISONES}

A lo largo de este artículo hemos pretendido destacar varios aspectos relacionados con la práctica del skate, tomando como base la investigación etnográfica que sobre este colectivo venimos realizando en la ciudad de Madrid. Primeramente, se ha explicado la metodología con la que decidimos llevar a cabo esta investigación, la cual hemos descrito como una etnografía "mixta" (Konizets, 2010) y "multisituada" (Marcus, 1995), dado que no se limita a la interacción cara a cara en un sólo lugar, sino que incluye interacción cara a cara y digital en varios lugares, tanto físicos (diversos barrios y espacios en Madrid y su área metropolitana) como digitales (diversos espacios digitales dentro de Internet).

Seguidamente, hemos analizado algunas de las características que definen al skate como práctica y su evolución a lo largo del tiempo hasta desembocar en el denominado "streetstyle", el estilo más conocido de skate y el responsable de

22 Las tecnologías virtuales son un complemento y no un sustituto de la actividad real. Esta es una de las cinco reglas de la virtualidad señaladas por Steve Woolgar (regla número 3) como una forma de problematizar las afirmaciones deterministas sobre los efectos e impactos de las nuevas tecnologías. Las otras reglas señaladas por Woolgar, que también son una respuesta empírica a las afirmaciones deterministas, son las siguientes: regla número 1: La aceptación y utilización de las nuevas tecnologías depende de forma crucial del contexto social local; regla número 2: Los miedos y riesgos asociados con las nuevas tecnologías están distribuidos socialmente de forma desigual; regla número 4: Cuanto más virtual, más real; y regla número 5: Cuanto más global, más local.

23 Otras interesantes iniciativas, proyectos y empresas en torno a la cultura skate en Madrid con una fuerte presencia en los medios digitales y las redes sociales son el colectivo 80Brigade, ya mencionado con anterioridad, (http://80brigade.blogspot.com.es; https://www. facebook.com/80brigade.org) junto a la escuela de skate y longboard Entabla (http://www. entabla.es; http://www.entabla.es/blog), o también las tiendas Welcome Skateboarding (http:// www.welcomesk8.com) y Dagger Family Shop (https://www.facebook.com/DaggerFamilyShop). 
que esta práctica se asocie fundamentalmente con la cultura urbana o callejera.

Posteriormente, hemos analizado algunas de las características que hacen del skate una subcultura y el tipo de elementos simbólicos que utiliza para manifestarse y reafirmarse y "marcar" así su diferencia con respecto a otras culturas y subculturas, aunque paradójicamente muestre un alto grado de diversidad intra-subcultural e incorpore elementos de varias de ellas. También hemos analizado los intentos y estrategias institucionales por "normalizar" y "domesticar" la práctica del skate, centrándonos en el caso paradigmático de los skateparks. Un tipo de instalación cuya construcción en diferentes ciudades y municipios, a instancia de las autoridades municipales, es una medida tendente a controlar y regular a los skaters, pero que a un tiempo está contribuyendo, quizá de forma no intencionada, a popularizarlo y al desarrollo y expansión de su práctica.

Asimismo, hemos señalado algunas contradicciones propias de la cultura skate actual. Durante nuestra observación de la práctica del skate en diversos espacios madrileños y nuestra interacción con diversos informantes, hemos identificado tendencias y dinámicas a primera vista contradictorias, pero que en su interdependencia dan un sentido propio y actual a esta práctica en la realidad actual. La práctica del skate hoy en día se caracteriza por una gran transversalidad y heterogeneidad de practicantes, espacios, estilos, gustos musicales, etc., que puede entrar en colisión con la tendencia a considerar esta práctica como una subcultura con identidad propia: podemos encontrar skaters de todas las edades y posiciones sociales, pertenecientes a diferentes generaciones y modos de entender esta práctica social, chicos o chicas y hombres o mujeres, -aunque el género masculino sigue siendo el predominante.${ }^{24}$ Asimismo, hay skaters que prefieren el streetstyle mientras que otros prefieren patinar y relacionarse en los skateparks y practicar el vertical, o que alternan diversos estilos y espacios. Otros lo entienden más como un deporte y otros utilizan la tabla simplemente como un medio de transporte con el que moverse por la ciudad. En lo musical, encontramos skaters muy influenciados por la cultura hip hop, que pintan graffitis y visten gorras y ropas holgadas, otros que escuchan música punk y hardcore, otros que prefieren el reggae y fuman marihuana, y otros que aglutinan diferentes aspectos de esta diversidad de influencias.

Por último, hemos querido destacar la importancia que juegan las tecnologías digitales en el skate actual, un aspecto que consideramos uno de

24 Así lo hemos observado durante nuestro trabajo de campo y lo han señalado algunos de nuestros informantes:

- Antes no había casi mujeres, ahora se están viendo más

- Hay una piba aquí que patina muy bien

- Dos o tres... (ENT-2)

En particular se ha observado un cambio de tendencia significativo respecto a principios de los 90 "cuando veías chicas en los skateparks, pero eran groupies, que iban a ver..., a ver si ligaban con algún skater y tal, pero ni patinaban. Ahora las ves que ya llevan su patín y ves que alguna ya patina, y que les gusta. Y eso está guay (ENT-3) 
los principales hallazgos de esta investigación. Las nuevas tecnologías digitales impulsadas por la sociedad de la información han aportado a los skaters nuevas formas de producir, distribuir, recibir y comentar su práctica, las cuales se suman a otras tecnologías tradicionales esenciales para su promoción como subcultura como fueron la fotografía y el vídeo. El skate actual es tanto físico como digital, pues tan importante como lo que ocurre en las calles y en los skateparks es lo que se distribuye y comenta en las redes digitales. Por lo tanto, creemos que cualquier investigación actual sobre este colectivo no debe ignorar el tipo de interacciones que se desarrollan en Internet, unas interacciones mediante los cuales los skaters extienden, reafirman y afianzan su sentido de identidad y comunidad.

La ciudad de Madrid se presenta actualmente como un espacio privilegiado para el estudio de todas estas cuestiones, ya que la práctica del skate está conociendo un considerable aumento de interés en la capital (o una nueva "oleada", por decirlo con las palabras de uno de nuestros informantes). Esta investigación se presenta, por tanto, como una primera respuesta al visible incremento de la práctica del skate en la ciudad Madrid, y esperamos que sus resultados sirvan para entender el estado actual del skate en esta ciudad y el significado de esta práctica en la sociedad contemporánea.

\section{BIBLIOGRAFÍA}

BEAL, B. (1995): "Discualifying the Oficial: An exploration of social resistance through the subculture of Skateboarding", Sociology of Sport Journal, vol. 12, pp.:252-267.

BELL, D. (1976): The coming of Post-Industrial Society. Basic Books, Inc. New York.

(1977): Las contradicciones culturales del capitalismo. Alianza Universidad. Madrid.

BORDEN, I. (2001): “Another Pavement, Another Beach: Skateboarding and the Performative Critique of Architecture", derived from Iain Borden (2001), Skateboarding, Space and the City: Architecture and the Body, Oxford, Berg Publisher. Disponible on-line: http://discovery.ucl.ac.uk/26049/1/ Borden Another Pavement.pdf

CAMINO, X. (2008): "Reinterpretando la ciudad: la cultura skater y las calles de Barcelona", en Puig y G. Maza (coords.) El deporte en los espacios públicos urbanos de Barcelona. Monográfico de Apunts. Educación Física y Deportes (91), Barcelona. Disponible on-line: http://redined.mecd.gob.es/xmlui/bitstream/ handle/11162/44764/01520123000261.pdf?sequence $=1$

(2012): Estudio cultural del Skateboarding en Barcelona (1975-2010). Tesis Doctoral, Universitat Rovira i Virgili (Tarragona), Departament d'Antropologia, Filosofia i Treball Social, Programa de Doctorado de Antropología Urbana. Disponible on-line: http://www.tdx.cat/bitstream/handle/10803/81714/TESI SK8. pdf? sequence $=1$

CLARKE, G. (1990): "Defending Ski-Jumpers: A Critique of Theories of Youth Subcultures $\mathbb{Q}$, in

S. Frith and A. Goodwin (eds) On Record: Rock, Pop and the Written Word, pp. 81-

97. London: Routledge. 
CLIFFORD, J. (2008): Itinerarios transculturales. Barcelona. Gedisa.

DE CERTEAU, M. (1988): The Practice of Everyday life. Berkeley. University of California Press.

DOUGLAS, M. (1988): Estilos de pensar. Ensayos críticos sobre el buen gusto, Gedisa, Barcelona.

FRITH, S. (1996): "Música e identidad", en HALL, S. y DU GAY, P. (comps.). Cuestiones de identidad cultural, Madrid, Amorrortu.

GOFFMAN, E. (1993). La presentación de la persona en la vida cotidiana. Buenos Aires.

Amorrortu.

GORDON, M. M. (1947): "The Concept of the Subculture and its Application», Social Forces, vol. 26(1), pp.: 40-42.

HAMMERSLEY, M., y ATKINSON, P. (2009). Etnografía. Métodos de investigación. Barcelona. Paidós.

HEBDIGE, D. (2004): Subcultura. El significado del estilo, Barcelona, Paidós.

HINE, Ch. (2004): Etnografía Virtual. Barcelona. Editorial UOC.

JANESICK, V. J. (2000). "The Choreography of Qualitative Research Design: Minuets, Improvisations, and Crystallization.” En Denzin, N.K., y Lincoln, Y. S. (eds.), Handbook of Qualitative Research, Thousand Oaks, CA. Sage Publications, pp. 379-399.

KOZINETS, R. (2010): Netnography: doing ethnographic research online. London, Sage.

LARAÑA, E. (1986): "Los nuevos deportes en las sociedades avanzadas", Revista de Occidente, $\mathrm{n}^{\circ}$ 62-63, pp: 5-22.

LÉVI-STRAUSS, C. (1970): El pensamiento salvaje. México. Fondo Cultura Económica.

MARCUS, G. E. (1995): "Ethnography in/of the World System: The Emergence of Multi-Sited Ethnography". Annual Review of Anthropology, 24, pp. 95-117.

OPPERMANN, M. (2000): «Triangulation - A Methodological discussion». International

Journal of Tourism Research. Vol. 2. N. 2. pp. 141-146.

REGUILLO CRUZ, R. (2000): Emergencia de culturas juveniles. Estrategias del desencanto, Argentina. Norma.

SIMMEL, G. (2000): «El conflicto de la cultura moderna», Revista Española de Investigaciones Sociológicas, $\mathrm{n}^{\circ}$ 89: pp.: 315-330.

THORNTON, S. (1995): Club cultures: music, media and subcultural capital. Cambridge, UK: Polity Press.

(1997): "Introduction". En Gelder, K, and Thornton, S. (eds.), The Subcultures Reader. London: Routledge, pp. 1-11.

WOOLGAR, S. (2005): "Cinco reglas de la virtualidad". En Woolgar, S. (ed.), ¿Sociedad virtual? Tecnología, ‘cibérbole’, realidad. Barcelona. Editorial UOC. 